\title{
Modified Maintenance Network Model for Urban Rail Transit Systems Based on the Variable Coverage Radius: Evidence from Changchun City in China
}

\author{
Yuan Jiang, ${ }^{1}$ Baofeng Sun $\mathbb{D}^{1},{ }^{1}$ Lili Zheng $\left(\mathbb{D},{ }^{1}\right.$ Wankun Cui, ${ }^{1}$ and Xinhua Mao ${ }^{2,3}$ \\ ${ }^{1}$ College of Transportation, Jilin University, Changchun 130022, China \\ ${ }^{2}$ School of Economics and Management, Chang'an University, Xi'an 710064, China \\ ${ }^{3}$ Department of Civil and Environmental Engineering, University of Waterloo, Waterloo N2L 3G1, Canada
}

Correspondence should be addressed to Lili Zheng; lilizheng@jlu.edu.cn

Received 16 September 2019; Revised 17 February 2020; Accepted 25 February 2020; Published 1 August 2020

Guest Editor: Maria C. Falvo

Copyright (c) 2020 Yuan Jiang et al. This is an open access article distributed under the Creative Commons Attribution License, which permits unrestricted use, distribution, and reproduction in any medium, provided the original work is properly cited.

Network-wide maintenance lacks strong theoretical support and practical cases. However, research on this topic has entered an extensive exploratory stage; for example, new network design methods are being sought, and successful practices from traditional maintenance by line and by profession are being incorporated. This paper proposes a novel set-covering model with the variable coverage radius for the maintenance network of urban rail transit systems in the context of network-wide maintenance. The concepts of network-wide maintenance follow principles that are similar to those of bio-geography-based optimization (BBO), i.e., patterns of migration, variation, and extinction of different populations in different habitats. Therefore, a BBO algorithm is implemented with combinatorial optimization programming. Experiments from Changchun city in China show that the proposed model and algorithm are effective in fulfilling network-wide requirements through a direct tradeoff between the coverage radius and maintenance response time. In addition, the maintenance capacity and variable coverage radius of each maintenance point influence both the maintenance timeliness and resource utilization of maintenance units.

\section{Introduction}

As of December 31, 2018, according to a statistical analysis of urban rail transit lines, 169 urban lines in 32 cities in mainland China were in operation, having a total length of 5494.9 kilometers. New lines with a length of 647.8 kilometers have created a record high. After the previous peak in construction in the past five years, the increasingly perfected rail transit network has entered a period of stable operation and maintenance. The first seminar on the topic of China's urban rail transit operation equipment maintenance innovation and practice was held in Shanghai in November 2015. The concept of "safe, reliable, and efficient operation of urban rail transit, with the maintenance guarantee as the top priority" was stated. In April 2016, the National Development and Reform Commission approved the founding of the first "National Engineering Laboratory for Urban Rail
Transit System Safety and Maintenance" in China, stressing that the issue of urban rail transit system maintenance is just as important as planning and construction. In June 2019, the 3 rd conference on vehicle operation and maintenance for Chinese urban rail transit systems was held by http://www. chinametro.net, focusing on security and intelligent and lean operation. Through those series of events, network-wide maintenance has been a very hot topic of discussion. In particular, based on the concept of network-wide maintenance, in contrast to the modes of maintenance by line and by profession, the problem of how to construct a robust maintenance network for urban rail transit networks arises, which is an important part of the health management of such networks.

The maintenance network is a complex network system consisting of maintenance demand points, maintenance points, resource supply points, and paths connecting these 
nodes, and it is an expansion network with service capabilities as the core. The urban rail transit maintenance network is a functionally complex network serving the urban rail transit physical network. As an implementation of network-wide maintenance, it consists of maintenance demand points, maintenance points, and maintenance paths, and it performs network operation maintenance and security protection.

In practice, the concept of network-wide maintenance comes from networked maintenance, which reflects the new demands of networked operation for the maintenance of urban rail transit systems:

(1) The failure of a certain rail or running vehicle in urban rail transit will affect not only the traffic and passenger transport organization of the line but also those of relevant lines, even causing faults over a larger scope that will lead to new emergencies. Due to the urgency of functional enhancement, the concept of network-wide maintenance has shifted from the strategic level to the tactical level.

(2) The efficiency of the traditional mode of maintenance by line needs to be improved. The modes of regional network maintenance and network-wide maintenance are more advantageous.

(3) The sharing of maintenance resources between different maintenance points is a necessary condition to ensure that maintenance resources are immediately available, and professional maintenance personnel are also included as maintenance resources for sharing and scheduling.

(4) The transfer stations are nodes that propagate faults with obviously high importance in network-wide maintenance, and more emphasis should be placed on them.

These aspects are the focus of line management, and they need standardized and sufficient maintenance units to provide protection.

Based on the concept of network-wide maintenance, maintenance network design for urban rail transit systems aims to divide the whole maintenance network into several regional subnets; allocate and optimize various maintenance resources for regional subnets, lay out maintenance points, units, and warehouses; and, ultimately, form a safe, reliable, and timely response and a low-cost, low-consumption maintenance network to ensure the safe operation of urban rail transit systems.

This paper studies urban rail transit maintenance network design based on the concept of network-wide maintenance. We organize this paper as follows. Section 1 proposes problems and improvement directions based on a literature review. Both Sections 1 and 2 define the new problem of network-wide maintenance and quantify important parameters. Basic assumptions such as coverage and guarantee rules are constructed, and a maintenance network design model for urban rail transit systems based on the variable coverage radius is established and developed. Section 3 designs the bio-geography-based optimization (BBO) algorithm; Section 4 applies the model and analyzes the sensitivity of impact factors. Section 5 presents the conclusions and future research directions.

\section{Literature Review}

In the academic field, the maintenance network design problem can be classified as a discrete multifacility location problem, and its theoretical model can be summarized as three classic models: the $P$-center model [1], the $P$-median model [2], and the set-covering model [3]. The set-covering model concerned in this paper is the most widely used in site selection or network location, and it is applicable to firefighting units, police stations, hospitals or emergency centers [4], telephone exchange centers, gas stations, and other research objects [5]. It covers a given level of demand with a minimum amount of facility or total construction cost, i.e., from a given set of alternatives, it chooses facility locations with the goal of minimizing costs based on certain coverage rules, minimizes the number of facilities, and maximizes profits or service. Farahani et al. offer a clear classification and review of the set-covering model for facility location and imply that the characteristics and functional location for maintenance points align with these goals just like normal facilities such as hospitals [6].

Berman et al. [7], Bai et al. [8], and Akella et al. [9] discussed the application of coverage models in site selection. A set-covering model using the variable coverage radius has obvious advantages in solving certain problems. Therefore, this type of model has been explored and developed very quickly in real-world practice. For instance, Berman et al. [10] achieved a direct tradeoff between the coverage radius $r$ and the facility variable cost $\varphi(r)$. Then, Berman and Krass emphasized three implicit assumptions for the set-covering model: full coverage or no coverage; individual coverage; and a fixed coverage radius [3]. They summarized the extensive research on the maximum covering model, progressive covering model, cooperative covering model, and variable radius covering model and concluded that the set-covering model with the variable coverage radius is more in line with actual needs. Bashiri and Fotouhi used the maximum-minimum method to determine the coverage radius under the assumption of the existence and robustness of the optimal solution [11].

In contrast to the direct tradeoff between the coverage radius and facility cost in [10] and the robustness of the solution in [11], Davari et al. considered that the coverage radius is determined by the farthest distance between the specified node and facility [12]. If speed is not considered, the farthest distance is equivalent to the transit time between nodes, and the coverage radius is converted into a function related to the transit time between nodes and their facility. Since the transit time is uncertain, it can be considered a fuzzy variable. Our research considers many more characteristics of the rail transit maintenance network and covers the radius equivalent to the farthest distance between the maintenance center and the point under maintenance. This distance is determined by the speed and transit time of the maintenance unit. The variable coverage radius can thus be 
converted into a function related to the response time of the maintenance point.

To solve set-covering models, in addition to classic heuristic algorithms such as genetic algorithms, the BBO algorithm, which is a type of new artificial intelligence algorithm, has achieved much more far-reaching applications. Based on natural bio-geography and its mathematics, Simon first proposed the BBO algorithm and then discussed how it can be used to solve optimization problems [13]. To test the performance of the $\mathrm{BBO}$ algorithm, he used 14 benchmark functions to compare with seven population-based optimization algorithms, such as genetic algorithms and evolutionary algorithms. The BBO algorithm was shown to be the most effective. In its updating mechanism, the $\mathrm{BBO}$ algorithm is different from other group intelligent optimization algorithms such as the hybrid teaching-learning algorithm; it simulates the behaviors of species migration and mutation in bio-geography and designs the operators of migration, mutation, and clearance. The operators simulate the migration, variation, and extinction of species communities in biogeography, and thus, they are strong in problem solving; they solve complex combinatorial optimization problems through individual collaboration and competition among groups. In problem solving, globally optimal solutions can be found faster compared to the use of traditional optimization algorithms. Therefore, we employ the $\mathrm{BBO}$ algorithm to solve the proposed model, paying more attention to make the heuristic method itself a suitable problem. Simon et al. followed this strategy to establish a multiobjective and heterogeneous indoor wireless network planning model and solved the model using the BBO algorithm [14].

We found three approaches for improving the $\mathrm{BBO}$ algorithm: (1) we can improve the original population emergence mechanism to enhance algorithm performance, e.g., Ergezer et al. proposed a new antithetic learning mechanism to increase the variety of populations [15]. (2) We can redesign the operators of migration, mutation, and clearance to expand the advantages of $\mathrm{BBO}$. For instance, $\mathrm{Ma}$ and Simon explored the behavior of six different migration models in $\mathrm{BBO}$ and investigated their performance by integrating a hybrid crossover of genetic algorithms together with $\mathrm{BBO}$ to change the migration operator, which is blended bio-geography-based optimization (B-BBO) [16]. (3) We can improve the searching capability of the algorithm, i.e., Shukla and Singh enabled searching and discovered excellent individuals by importing gradient search and grid search methods into BBO [17]. In this paper, we prefer the second approach and redesign the operators to make them exactly fit the set-covering model established and the network-wide maintenance decision-making procedure we defined.

\section{Modeling Methodology}

In this paper, we explore improving the classic set-covering model to construct a reliable and quick-response maintenance network for urban rail transit networks as follows:
(1) The demand for each maintenance demand point of the maintenance network for urban rail transit systems is different. The service capability of each maintenance point can be either a standard service capability (same) or a hierarchical service capability (differentiated) to acclimate to changes in demand and supply. To that end, to distinguish the importance of maintenance demand points, employing the variable coverage radius to cover distinct maintenance points is more targeted and adaptable.

(2) In selecting the location of maintenance points, a quick response after problems is the primary technical and safety requirement, compared to the tradeoff between the coverage radius and cost. For this reason, the coverage radius of each maintenance point depends on its effective response. In this paper, we directly weigh between the coverage radius and the maintenance response time, and the actual response time does not exceed the timely response time.

(3) The coverage and rules for maintenance points are proposed and developed. Each maintenance point is responsible for both nodes (station) and lines (tracks). The areas of responsibility of all points are mutually exclusive. Different maintenance points and their units are selected and configured according to the types of faults.

3.1. Problem Statements. The maintenance demand point is the protection object of the maintenance network. In principle, all nodes and edges of the maintenance network are maintenance demand points, i.e., vehicles and rail tracks, thereby forming a set of maintenance demand points $g f$ (station set $V$ and track set $E$ ), or $G=\{V, E\}$. According to the type of station, set $V$ is subdivided into three subsets: stop stations $\left(V_{1}\right)$, same-way transfer stations $\left(V_{2}\right)$, and multimodal transfer stations $\left(V_{3}\right)$. The stations in $V$ are denoted as $v_{\mathrm{ni}}(n=1,2,3)$, and the next station of station $i$ is denoted by $k$; the track between these stations in set $E$ is denoted as $e_{\mathrm{ik}}$. Vehicles must be moved to the next station for repair, while tracks require on-site repair.

The set of stations as maintenance demand points $V$ is also the set of alternative maintenance points. The set of maintenance points refers to the set of selected maintenance demand points, denoted as $V ı$. The distance of the maintenance unit moving between a maintenance point $v_{j}^{\prime}$ and the maintenance demand point $f$ in the maintenance network is denoted as $L_{\mathrm{fi}}$, and their collection is defined as set Path $=\left\{L_{f j}(f, j)\right\}$. Together, the maintenance points and paths constitute the maintenance network $G \prime=\{V I$, Path $\}$. Once a fault occurs in an urban rail transit system, it is necessary for the maintenance unit and repair parts to arrive in a timely manner and for the fault to be handled. This process is completed by the maintenance point.

The idea behind designing a maintenance network model for urban rail transit systems based on the set-covering model is as follows: first, we determine the repair 
demand stations set $V$; then, by changing the coverage radius $R_{j}$, according to the coverage and rules of maintenance points $g f$ established in this paper, we determine how the demand points can be covered. The set of maintenance points, together with the maintenance demand point $f$ that can be covered by the maintenance point $A(j)$, establishes the urban rail transit maintenance network 0-1 integer programming model $B(f)$; thus, it meets dual goals of the minimum number of maintenance points and shortest total maintenance path.

\subsection{Model Hypotheses}

Hypothesis 1. An urban rail transit network is divided into several exclusive subareas called planned cells. The stations and tracks in each planned cell are assigned to a maintenance point. The relevant planned cells are determined as the coverage of this point.

Hypothesis 2. The requirements for all maintenance demand points are the same, the demand for each maintenance demand point is provided exclusively by one maintenance point, and there is no service overlap.

Hypothesis 3. The stations are location alternatives for setting up maintenance points.

Hypothesis 4. The number of maintenance points and the allocation plan for each specific maintenance point are uncertain; we constrain the service capacity of each maintenance point, and calculation is performed under these assumptions.

Hypothesis 5. The service capacity of a maintenance point is bound by its own mobile maintenance units, and the number of initial maintenance units for each maintenance point is the same.

Hypothesis 6. Since stations are closely related to tracks, if the maintenance demand station $v_{\mathrm{ni}}(n=1,2,3)$ is assigned to a maintenance point $v_{j}^{\prime}$ for maintenance, track $L_{\mathrm{fj}}$ on the path is also assigned to the same point $v_{j}^{\prime}$.

Hypothesis 7. The repair parts inventory of each maintenance point is highly shared. If the repair parts at a maintenance point are insufficient, the nearest maintenance point should supply them in time, and the spare parts inventory should be replenished in a timely manner.

Hypothesis 8. A faulty vehicle can be driven to the next station on its route at any time or can be transported to a maintenance point in a timely manner.

3.3. Notations. The notations used in this section are listed as follows:

Parameter:

$t^{\prime}$ Response time to a breakdown $t$ Actual response time

$t_{0}$ Unit repair time

$t_{1}$ Mobility time

$t_{2}$ Repair time

$t_{3}$ Travel time

$R_{j}$ Coverage radius of maintenance support point $j$

$v_{0}$ Mobile speed maintenance unit moving speed

$q_{\mathrm{fj}}$ Maintenance demand system

$f$ The number of mobile maintenance support units actually needed

$Q_{\mathrm{jr}}$ The number of mobile maintenance support units actually needed for maintenance support point $j$

$Q_{j}$ Maintenance support point $j$ maintenance support unit configuration number

$Q_{\text {max }}$ Service support point $j$ service capability

$M$ Number of rail stations

Decision variables (Binary):

$X_{j}$ If $v_{j}^{\prime}$ is selected to establish the maintenance support point, $X_{j}=1$; otherwise, 0

$Y_{\mathrm{fj}}$ If $v_{j}^{\prime}$ provides the maintenance actions for maintenance demand system $g f, Y_{\mathrm{fj}}=1$; otherwise, 0

$Y_{\mathrm{kj}}$ If $v_{j}^{\prime}$ provides the maintenance actions for the vehicles of rail station $k, Y_{\mathrm{kj}}=1$; otherwise, 0

$Y_{\mathrm{ikj}}$ If $v_{j}^{\prime}$ provides the maintenance actions for rail $e_{\mathrm{ik}}$, $Y_{\mathrm{ikj}}=1$; otherwise, 0 .

\subsection{Descriptions of Important Parameters}

(1) We differentiate the importance of maintenance demand points. The coverage of maintenance points is differentiated by a variable coverage radius. For $V_{1}$, $V_{2}$, and $V_{3}$, there is the following:

$$
R_{j}=\left\{\begin{array}{l}
\eta R, \quad j \in V_{1}, \eta \in(1,2), \\
R, \quad j \in V_{2}, \\
\varphi R, \quad j \in V_{3}, \varphi \in(0,1) .
\end{array}\right.
$$

Here, $R$ is the average coverage radius of maintenance points within the maintenance network. The elastic coefficients $\eta$ and $\varphi$ of $R_{j}$ are rooted on two main influence factors in theory, i.e., the urban rail transit network size and the average spacing between stations. In our research, we take the latter as reference because, in practice, the average spacing is one of the key design parameters for urban rail transit network. Here, $\eta$ and $\varphi$ of their threshold values are estimated through Changchun case and other similar medium size cities such as Shenyang and Haerbin.

If the coverage radius $R_{j}$ is small, the support coverage is correspondingly small, which means that more maintenance points are required to meet the 
maintenance demand, increasing the construction cost. Conversely, if $R_{j}$ is large, fewer maintenance points are required, the construction cost is lower, but the response time performance in long-distance faults can be poor.

(2) The deployment of maintenance units

Maintenance units are mainly divided into two types: mobile and stationary. Mobile maintenance units handle both vehicle and rail faults, while stationary maintenance units handle only vehicle faults. In the case of vehicle faults, the corresponding maintenance point is notified, and the vehicle is driven to the next station on its route; maintenance is carried out by both mobile and stationary maintenance units. In the case of rail faults, mobile maintenance units are sent out to perform maintenance.

Within the actual coverage of maintenance support point $v_{j}^{\prime}$, the number of maintenance support units, $Q_{j}$, should be more than the actual demand, $Q_{j r^{\prime}}$, and should not exceed the service capacity, $Q_{j \max }$, which is formulated as follows:

$$
Q_{j r} \leq Q_{j} \leq Q_{\text {jmax }}
$$

(3) The rules of resource allocation for maintenance demand stations $v_{\text {ni }}(n=1,2,3)$ are as follows:

For the three types of stations, $V 1, V 2$, and $V 3$, the value of the maintenance resource property factor $\alpha_{i}$ is different in consideration of station function type and design capacity.

$$
\alpha_{i}= \begin{cases}1, & i \in V_{1}, \\ 2, & i \in V_{2}, \\ 4, & i \in V_{3} .\end{cases}
$$

When a new line passes $v_{\text {ni }}(n=1,2,3)$, i.e., considering the complex effect of line increase, when the number of lines $\beta_{i}$ increases by one, the number of its preassigned maintenance units, $q^{\text {imax }}$, increases by 0.5 standard units, $q^{\max }$ :

$$
q^{\operatorname{imax}}=\left\{1+0.5 \alpha_{i}\left(\beta_{i}-1\right)\right\} \cdot q^{\max } .
$$

(4) Coverage radius $R_{j}$ for maintenance point $j$

The actual response time $t$ includes maneuver time $t_{1}$, maintenance time $t_{2}$, and maintenance vehicle time on trip $t_{3}$; thus, $t=t_{1}+t_{2}+t_{3}$. To meet the timely response requirement, the actual response time must be less than or equal to the expected response time, i.e., $t \leq t^{\prime}$. The maximum excepted response time $t /$ is the minimum requirement for the maintenance time.
If the traveling speed of maintenance units is $v^{0}$, the relationship among the distance between maintenance demand point $g f$ and maintenance point $v_{j}^{\prime}$ (denoted as $\left.L_{\mathrm{fj}}\right), t^{\prime}$, and $v^{0}$ is as follows:

$$
L_{\mathrm{fj}}=\left(t^{\prime}-t_{1}-t_{2}\right) \cdot v^{0} .
$$

According to the set-covering model, the relationship between the maintenance point $v_{j}^{\prime}$ s coverage radius $R_{j}$ and $L_{\mathrm{fj}}$ is as follows:

$$
R_{j}=\max \left\{L_{f j}\right\}=\max \left\{\left(t^{\prime}-t_{1}-t_{2}\right) \cdot v^{0}\right\} .
$$

When $v^{0}$ is constant, $t^{\prime}$ determines the value of $R_{j}$. To ensure a timely response capacity, $t^{\prime}$ and $R_{j}$ can be interconverted according to the needs of the study. To simplify the calculation, the shortest fault maintenance time is defined as a unit maintenance time $t_{0}$; thus, the maintenance time of other faults can be defined as a multiple of the unit maintenance time.

$$
t_{2}=s \cdot t_{0}, \quad s \in N
$$

3.5. Objective Functions and Constraints. The objective functions and constraints of the maintenance network design model for urban rail transit systems (referred to as the design model) proposed in this paper are as follows:

$$
\begin{gathered}
\min \sum_{j \in V_{1}} X_{j}, \\
\min \sum_{j} \sum_{f} Y_{\mathrm{fj}} \cdot L_{\mathrm{fj}} .
\end{gathered}
$$

Subject to the constraints

$$
\begin{gathered}
Y_{\mathrm{fj}} \cdot L_{\mathrm{fj}} \leq R_{f}, \\
\sum_{j} \sum_{f} Y_{\mathrm{fj}} \cdot q_{\mathrm{fj}} \leq Q_{\mathrm{jmax}}, \\
\sum_{j \in V^{\prime}} Y_{\mathrm{ikj}}=1, \quad \forall i, k \in V, \\
\sum_{j \in V^{\prime}} Y_{\mathrm{kj}}=1, \quad \forall i, k \in V, \\
Y_{\mathrm{kj}} \leq X_{j}, \quad k \in V, j \in V^{\prime}, \\
Y_{\mathrm{ikj}} \leq X_{j}, \quad \forall i, k \in V, j \in V^{\prime}, \\
\sum_{j \in V^{\prime}} X_{j} \leq m, \\
X_{j} \in\{0,1\}, j \in 3 V^{\prime},
\end{gathered}
$$




$$
\begin{gathered}
Y_{\mathrm{ikj}} \in\{0,1\}, \quad k \in V, j \in V^{\prime}, \\
Y_{\mathrm{ikj}} \in\{0,1\}, i, \quad k \in V, j \in V^{\prime} .
\end{gathered}
$$

Equation (8) is the objective function of the model; it aims to ensure the minimum number of maintenance support points, which means the minimum construction cost. As another objective function of the model, equation (9) ensures the shortest total maintenance support distance that can achieve a timely response of the maintenance support network. Equation (10) is the constraint of the selection of maintenance support routes; based on this constraint, every single maintenance support route selected should be within the coverage of maintenance support point $v_{j}^{\prime}$, i.e., the distance $L_{\mathrm{fj}}$ between $g f$ and $v_{j}^{\prime}$ should be no more than the coverage radius $R_{\mathrm{j}}$ of $v_{j}^{\prime}$. Equation (11) is the service capacity constraint of the maintenance support point. The total maintenance demands assigned to the maintenance support point $v_{j}^{\prime}$ cannot exceed its service capacity $Q_{j \max }$. Equations (12) and (13) indicate that every single rail $e_{\mathrm{ik}}$ or rail station $v_{n i}(n=1,2,3)$ accepts service from only one maintenance support point $v_{j}^{\prime}$. Equations (14) and (15) indicate that only when alternative location $j$ is selected as a maintenance support point can it provide maintenance actions for rail station $k$; additionally, only when alternative location $v_{j}^{\prime}$ is selected as a maintenance support point can it provide support for the rails on route $L_{\mathrm{ff}}$. Equation (16) presents the constraint on the number of maintenance support points, which means that the number of maintenance support points cannot exceed the number of rail stations because the maintenance support points are located at the rail stations. Equations (17)(19) are all 0-1 decision variables.

\section{BBO Algorithm Design}

This research uses the $\mathrm{BBO}$ algorithm to solve the model proposed above. The $\mathrm{BBO}$ algorithm designs the migration operator, mutation operator, and clearance operator to simulate the migration of species communities between habitats, species variation, and species extinction in biogeography, and it solves complex combinatorial optimization problems through individual collaboration and competition among groups. Compared to other algorithms, the $\mathrm{BBO}$ algorithm has an advantage in its updating mechanism and global searching ability. Regarding the model in this research, the $\mathrm{BBO}$ algorithm is defined as follows.

In an urban rail transit maintenance support network, we define the maintenance demand of vehicles and rails as a collection of species and denote the maintenance support points as islands that are colonies of species. According to the principle of the algorithm, maintenance support points with more maintenance resources are more attractive to vehicles and rails with maintenance demands, i.e., these maintenance support points have a higher adaptability index. By adjusting the immigration rate $\lambda_{j}$, emigration rate $\mu_{j}$, and adaptation index $\mathrm{HSI}_{j}$, we identify the relationship between the maintenance support point and vehicles and rails. The mutation operator is introduced to make the maintenance network more stable and robust, and finally, the optimal solution is obtained.

We define the adaptation index $\mathrm{HSI}_{j}$ as the ratio of the number of rail stations and rails $Q_{j \mathrm{r}}$ supported by $v_{j}^{\prime}$ up to their max number $Q_{\text {jmax }}$, which is formulated as follows:

$$
\mathrm{HSI}_{j}=\frac{Q_{j r}}{Q_{j \max }}
$$

(1) When no maintenance support points have maintenance tasks, i.e., the number of rail stations $s$ and the number of railroad tracks $n$ that need maintenance are both 0 , the emigration rate is 0 , and the immigration rate $\lambda_{j}$ is the highest, which is denoted as $I$. We can formulate $\lambda_{j}$ as follows:

$$
\lambda_{j}=I \cdot\left(1-\frac{s}{n}\right)=I-\frac{Q_{\mathrm{jr}}}{Q_{\mathrm{jmax}}} .
$$

(2) As the maintenance demands increase, the maintenance of more vehicles and rails will be assigned to other maintenance support points, leading to an increase in the emigration rate $\mu_{j}$. Meanwhile, the available maintenance support resources will decrease, which will reduce the immigration rate $\lambda_{j}$.

(3) When the maintenance resources of a maintenance support point are fully utilized, i.e., when the number of stations and rails served by a maintenance support point is its maximum service capacity $Q_{j \max }$, the emigration rate is the highest, which is denoted as $E$, and the immigration rate $\mu_{j}$ drops to $0 . \mu_{j}$ can be formulated as follows:

$$
\mu_{j}=\frac{E_{s}}{n}=\frac{Q_{\mathrm{jr}}}{Q_{\mathrm{jmax}}} .
$$

(4) When the service capacity of $v_{j}^{\prime}$ and the number of stations and rails supported reach a balance, their relationship tends to be stable. To avoid local stability rather than global stability, the mutation operator $\alpha$ is used to obtain the global optimal solution. We set $\alpha=0.0005$.

The process of the algorithm is shown in Figure 1.

\section{Numerical Examples}

5.1. Urban Rail Transit Network and Basic Data. A case study using the urban rail transit operation network in Changchun, the capital city of Jilin Province in northeastern China, is employed to test the model. Compared with other same medium-sized cities in China, Changchun urban rail transit network steps in fast-speed developing stage and focuses on improving the accessibility between districts to solve the problem of difficult travel for the masses in the surrounding areas of the city. Its maintenance experience at present is not rich and lacks targeted solution for such a kind of network. Its maintenance network design thereby 


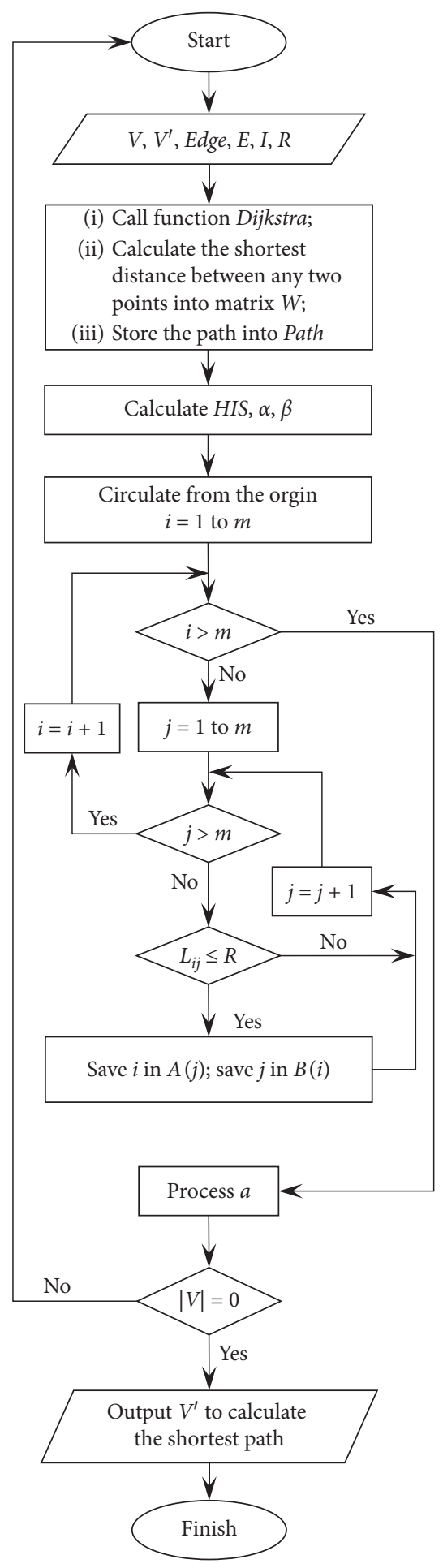

(1) Shortest path selection

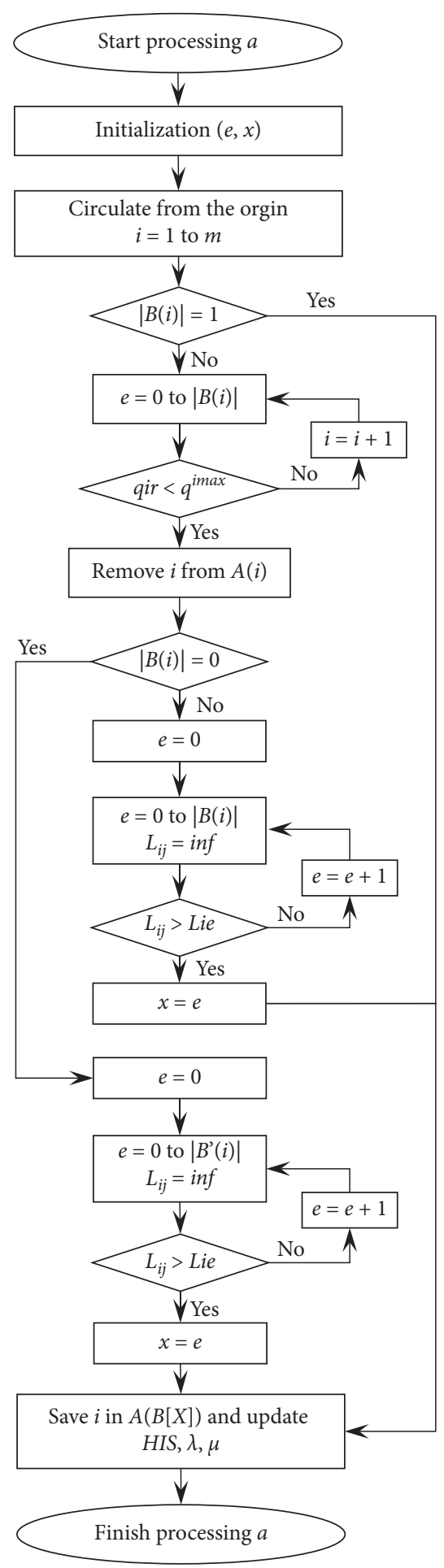

(2) Set-coverage model

Figure 1: Algorithm flow chart.

comes to one of the essential decisions of operation safety management.

Figure 2 shows the layout of the network, which consists of 4 lines, i.e., line 1 , line 2 , line 3 , and line 4 , and 77 rail stations, including 8 interchange stations. The 77 rail stations are the alternatives of the maintenance support points. We number the 77 rail stations as shown in Figure 2. We define $v_{2}=\{4,5,8,12,28,34\}$ and $v_{3}=\{23,53\}$; additionally, the remaining rail stations belong to $v_{1}$. Accordingly, we code the rails and collect the length data of each rail segment 

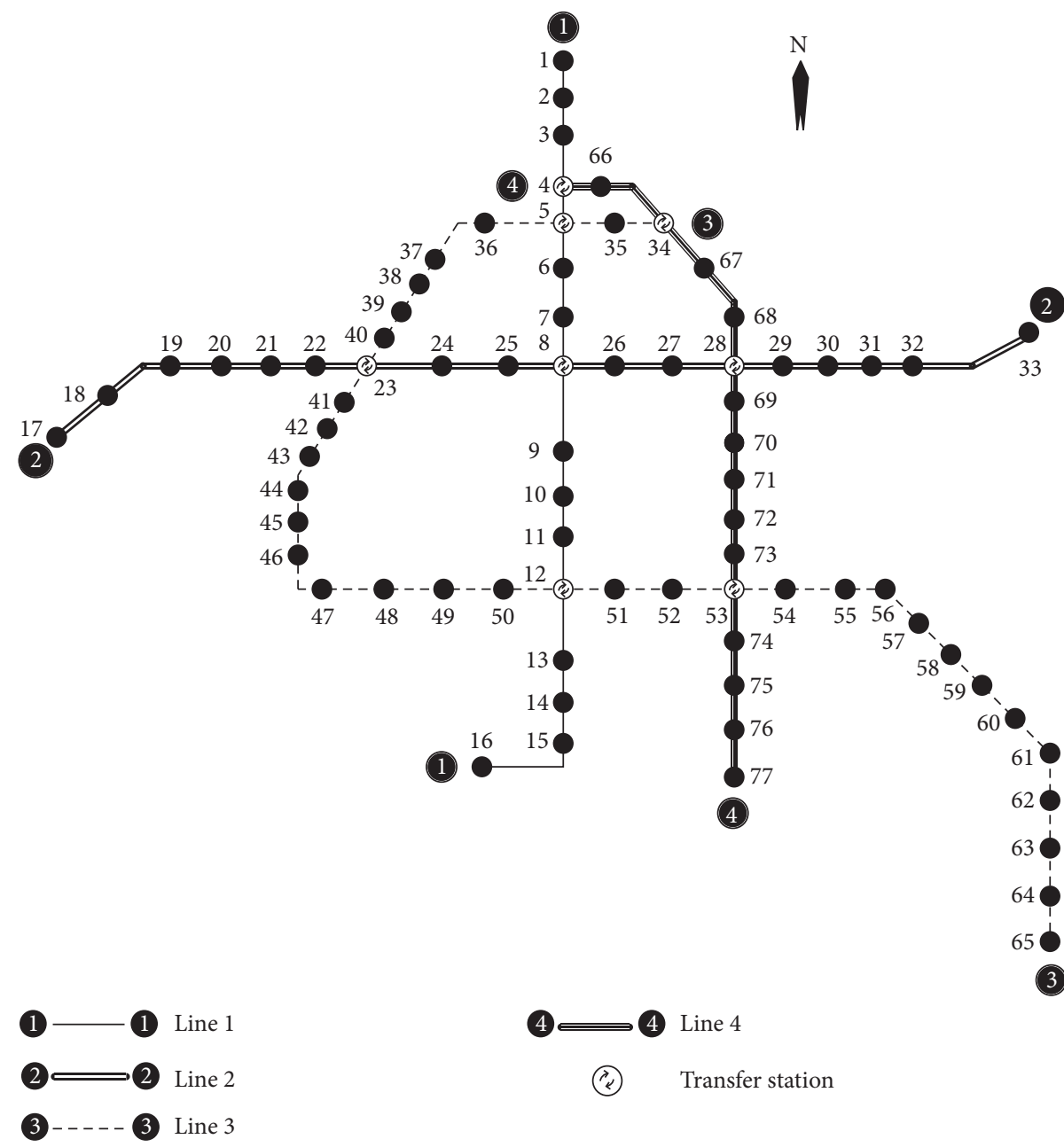

FiguRe 2: Layout of the urban rail transit network.

TABLE 1: Maintenance support points and their coverages in basic scenario.

\begin{tabular}{lcccc}
\hline$v_{j}^{\prime}$ & Number & $\begin{array}{c}v_{\mathrm{ni}}=(1,2,3) \\
\text { Code of rail stations }\end{array}$ & Number & $\begin{array}{c}e_{\mathrm{ik}} \\
\text { Code of rail stations }\end{array}$ \\
\hline 6 & 12 & $1,2,3,4,5,6,7,8,26,34,35,66$ & 14 & $1,2,3,4,5,6,7,8,25,33,34,35,67,68$ \\
12 & 11 & $9,10,11,12,13,14,15,16,48,49,50$ & 11 & $9,10,11,12,13,14,15,48,49,50,51$ \\
21 & 11 & $17,18,19,20,21,22,36,37,38,39,40$ & 11 & $16,17,18,19,20,21,36,37,38,39,40$ \\
29 & 12 & $27,28,29,30,31,32,33,67,68,69,70,71$ & 13 & $26,27,28,29,30,31,32,69,70,71,72,73,74$ \\
41 & 10 & $23,24,25,41,42,43,44,45,46,47$ & 10 & 14 \\
53 & 10 & $51,52,53,54,72,73,74,75,76,77$ & 12 & $52,53,54,55,56,75,76,77,78,79,80,81$ \\
60 & 11 & $55,56,57,58,59,60,61,62,63,64,65$ & 10 & $57,58,59,60,61,62,63,64,65,66$ \\
Total & 77 & & 81 & 13 \\
\hline
\end{tabular}

TABLe 2: Average lengths of the maintenance paths and arrival times.

\begin{tabular}{lcccc}
\hline$v_{j}^{\prime}$ & $v_{\mathrm{ni}}=(1,2,3)$ & $L(\mathrm{~km})$ & $\bar{L}(\mathrm{~km})$ & $\bar{t}(\mathrm{~km})$ \\
\hline 6 & 12 & 37.90 & 3.16 & 2.96 \\
12 & 11 & 32.50 & 4.15 & \\
21 & 11 & 45.60 & 3.13 & 2.98 \\
29 & 12 & 37.50 & 3.13 & 2.09 \\
41 & 10 & 31.30 & 2.35 & 2.09 \\
53 & 10 & 23.50 & 3.73 & 1.57 \\
60 & 11 & 41.00 & 3.23 & 2.49 \\
Total & 77 & 249.30 & 2.16 \\
\hline
\end{tabular}


between two adjacent rail stations. Case data here come from https://dt.8684.cn/cc_intro.

5.2. Model Optimal Solution. In the optimal solutions, all demand nodes are fulfilled, as shown in Tables 1 and 2, which is the basis of scenario analysis. Take maintenance support point 29 as an example. The service coverage of a single point is shown in Figure 3.

In this basic scenario, $Q_{\max }=15$ and the coverage radius, $\left(R_{1}, R_{2}, R_{3}\right)=(7.5,5,2.5)$, is abbreviated as $7.5 / 5 / 2.5 \mathrm{~km}$.

The analysis and revision of the optimal solution are as follows:

(1) The numbers of stations and rails served by each maintenance point are 11 and 11.5, respectively; the actual numbers of stations and rails served by each point are 11-12 and 10-14, respectively. Maintenance demand is averagely distributed, and any maintenance point is bound not to be too idle or congested.

(2) According to the coverage rules, demand point 23 is under the coverage of maintenance point 21 . However, since demand point 23 is a transfer station and its coverage radius requirement is $2.5 \mathrm{~km}$, point 21 cannot provide support for it, and it needs to look for other support points. Revision is required to ensure the timeliness of maintenance, causing this maintenance point to shift, but it is still consistent with the model's basic assumptions and solution conditions.

(3) Due to the large coverage radius $(7.5 \mathrm{~km})$ of the edge stop station, setting up a maintenance point (60) at the end of route 3 can support multiple demand points and avoid the addition of multiple maintenance points with a small coverage radius.

5.3. Performance Analysis. Performance experiment 1: in scenario $1, Q_{\max }=12,13,14,16,17,18$ and $\left(R_{1}, R_{2}, R_{3}\right)=$ $(7.5,5,2.5)$, abbreviated as $7.5 / 5 / 2.5$. The objective of the experiment is to examine the impact of changes in maintenance point $v_{j}^{\prime \prime} \mathrm{s}$ service capacity $Q_{\max }=$ $12,13,14,16,17,18$ on the maintenance network.

Performance experiment 2: in scenario $2, Q_{\max }=15$ and the coverage range is $8 / 5 / 2 \mathrm{~km}, 7.5 / 5 / 2.5 \mathrm{~km}, 7 / 5 / 3 \mathrm{~km}, 6 / 5 /$ $4 \mathrm{~km}$, and $5 / 5 / 5 \mathrm{~km}$. The objective of the experiment is to examine the sensitivity of changes in $R_{j}$ to the optimal solution to the design model, given a fixed $Q_{\text {jmax }}$. This examination also reveals the impact of the maintenance timeliness constraint on the maintenance network program.

Under various $Q_{j \max }$ and $R_{j}$ settings, the optimal solutions to the design model can be summarized in Tables 3 and 4.

For performance experiment 1 , the sensitivity analysis of $Q_{\text {jmax }}$ is as follows:

(1) Under the scenarios of Tables 1 and 3, the number of demand points served by each maintenance point is not averagely distributed and underperforms the basic scenario. For example, maintenance point 19 serves only 5 stations and 5 rails, with 6 maintenance

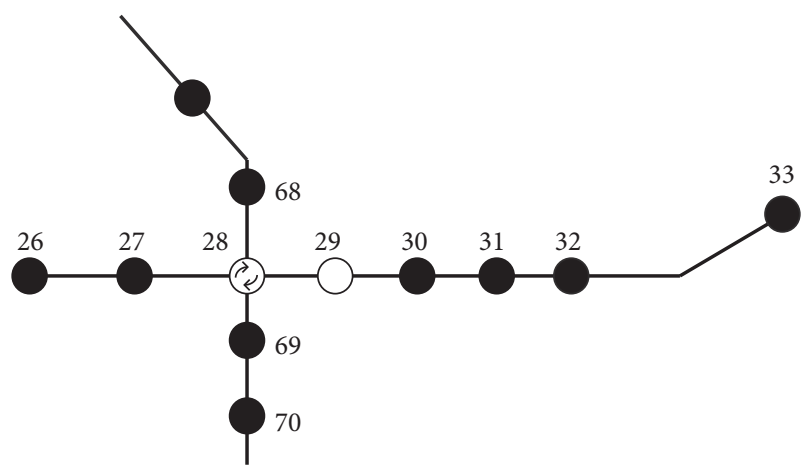

Maintenance support point
Maintenance coverage (station)
Maintenance coverage (rail)

FIgURE 3: Maintenance support point 29 and its coverage.

units needed; nonetheless, maintenance point 41 serves 16 stations and 16 rails, with 20 maintenance units needed. If the maintenance points are built according to a unified standard, this gap in actual demand will inevitably result in a large number of idle maintenance units.

(2) Under the scenario of Table 4, when $Q_{j \max }$ 's service capacity increases from 12 units to 18 , the number of maintenance points decreases from 11 to 7 and remains unchanged thereafter. The continuous increase in the number of maintenance points does not necessarily cause a decrease in the number of maintenance points or an increase in the total length of support routes. Additionally, the standard deviations of the support routes' average length $\bar{L}$ and average arrival time $\bar{t}$ are 0.072 and 0.055 , respectively. The change is relatively small, and the timeliness of maintenance can be fully achieved.

For performance experiment 2 , the sensitivity analysis of $R_{j}$ is shown in Table 5 as follows:

(1) In the fixed coverage radius of 5/5/5, 11 maintenance points are allocated, and the average response time is 2.26 minutes; 165 maintenance units are required. The utilization rate is $58.79 \%$. These indicators are the worst in all scenarios.

(2) Most of the maintenance points with the smallest coverage are located at the end of the line. Due to the fixed coverage radius, to ensure the timeliness of maintenance, two maintenance points are needed for stations 55-65 at the end of the line. Compared to the $7.5 / 5 / 2.5$ plan, an extra maintenance point is needed.

(3) Regardless of the variable coverage radius or the fixed coverage radius settings, the actual demands for maintenance units $Q_{\mathrm{jr}}$ of the five settings all fall between $[95,97]$. The actual demands are insensitive to the range of the radius $R_{1} / R_{2} / R_{3}$.

(4) The number of maintenance units $Q_{j}$ of the five comparison settings increases in turn, accompanied 
TABLE 3: Simulation results in scenario 1.

\begin{tabular}{lcccc}
\hline$v_{j}^{\prime}$ & Number & $\begin{array}{c}v_{\mathrm{ni}}=(1,2,3) \\
\text { Code of rail stations }\end{array}$ & Number & \multicolumn{1}{c}{$\begin{array}{c}e_{\mathrm{ik}} \\
\text { Code of rail stations }\end{array}$} \\
\hline 5 & 13 & $1,2,3,4,5,6,7,8,34,35,36,37,66$ & 14 & $1,2,3,4,5,6,7,33,34,35,36,37,67,68$ \\
12 & 12 & $9,10,11,12,13,14,15,16,48,49,50,51$ & 13 & $8,9,10,11,12,13,14,15,48,49,50,51,52$ \\
19 & 5 & $17,18,19,20,21$ & 5 & $16,17,18,19,20$ \\
29 & 11 & $28,29,30,31,32,33,67,68,69,70,71$ & 12 & 15 \\
41 & 16 & $22,23,24,25,26,27,38,39,40,41,42,43,44,45,46,47$ & 16 & $21,22,23,24,25,26,38,39,40,41,42,43,44,45,46,47$ \\
52 & 10 & $52,53,54,55,72,73,74,75,76,77$ & 10 & $53,54,55,56,76,77,78,79,80,81$ \\
60 & 10 & $56,57,58,59,60,61,62,63,64,65$ & 10 & $57,58,59,60,61,62,63,64,65,66$ \\
Total & 77 & & 81 & 12 \\
\hline
\end{tabular}

TABLE 4: Impact of different values of $Q_{j \max }$ on model solutions.

\begin{tabular}{|c|c|c|c|c|c|c|c|}
\hline$Q_{\text {jmax }}$ & $v_{j}^{\prime}$ & $L(\mathrm{~km})$ & $\bar{L}(\mathrm{~km})$ & $\bar{t}(\mathrm{~km})$ & $Q_{\mathrm{jr}}$ & $Q_{j}$ & Utilization rate (\%) \\
\hline 12 & 11 & 255.2 & 3.32 & 2.22 & 100 & 132 & 75.76 \\
\hline 13 & 10 & 255.4 & 3.32 & 2.22 & 99 & 130 & 76.16 \\
\hline 14 & 8 & 252.6 & 3.28 & 2.19 & 97 & 112 & 86.61 \\
\hline 15 & 7 & 249.3 & 3.24 & 2.16 & 96 & 105 & 91.43 \\
\hline 16 & 7 & 251.7 & 3.27 & 2.18 & 96 & 112 & 85.72 \\
\hline 17 & 7 & 253.4 & 3.30 & 2.20 & 95 & 119 & 79.84 \\
\hline 18 & 7 & 252.9 & 3.29 & 2.20 & 95 & 126 & 75.40 \\
\hline+ inf & 7 & 251.1 & 3.27 & 2.18 & 95 & 140 & 67.86 \\
\hline
\end{tabular}

TABLE 5: Simulation results in scenario 2.

\begin{tabular}{lcccccccc}
\hline$R_{1} / R_{2} / R_{3}(\mathrm{~km})$ & $v_{j}^{\prime}$ & $L(\mathrm{~km})$ & $\bar{L}(\mathrm{~km})$ & $T(\min )$ & $Q_{\mathrm{jr}}$ & $Q_{\text {jmax }}$ & $Q_{j}$ & Utilization rate $(\%)$ \\
\hline $8 / 5 / 2$ & 7 & 255.8 & 3.33 & 2.22 & 95 & 15 & 105 & 90.48 \\
$7.5 / 5 / 2.5$ & 7 & 249.3 & 3.24 & 2.16 & 96 & 15 & 105 & 91.43 \\
$7 / 5 / 3$ & 7 & 250.1 & 3.25 & 2.17 & 96 & 15 & 120 & 80.00 \\
$6 / 5 / 4$ & 8 & 251.6 & 3.23 & 2.16 & 97 & 15 & 150 & 64.67 \\
$5 / 5 / 5$ & 11 & 248.9 & 3.38 & 2.26 & 97 & 15 & 165 & 58.79 \\
\hline
\end{tabular}

by a decrease in the utilization rate from $90.48 \%$ to $58.79 \%$. The maintenance capacity and construction cost of the maintenance points are sensitive to the range change of the radius $R_{1} / R_{2} / R_{3}$.

\section{Conclusions}

The maintenance network of urban rail transit systems serves the physical network of urban rail transit. Through the scientific deployment of maintenance points, maintenance units, and maintenance part warehouses and the optimization of the configuration of various maintenance resources, we can obtain a safe, reliable, and timely response and a low-cost system, guaranteeing the safe operation of the urban rail transit system. In this paper, we study the design of a maintenance network for urban rail transit systems to provide methodological support for the construction and improvement of these systems. This paper obtains the following results:

(1) The number of maintenance units for maintenance points (the service capacity for maintenance points) should be considered. Additionally, the upper limit of the service capacity for maintenance points should be set. The layout plan for the maintenance network should also be obtained. Furthermore, the demand points and support points should be evenly distributed. Maintenance requirements and guarantees should be fulfilled as well. Finally, the ability of individual maintenance points to match each other will not cause congestion or idleness.

(2) The network-wide maintenance solution obtained by the variable coverage radius can not only meet the maintenance requirements but also set a larger coverage radius for areas where maintenance needs are dispersed, thereby reducing the number of maintenance points.

(3) Although in the fixed coverage radius solution, the maintenance barrier network layout setting can also meet the maintenance requirements, more maintenance points are set because the demand distribution in the network is relatively scattered, which increases the construction cost. The above two points show that the set-coverage model with the variable coverage radius is advantageous in solving the problem, and the obtained network arrangement is more reasonable and feasible.

(4) When constructing the maintenance network, we need to balance between the farthest service distance of maintenance points and the number of maintenance resource nodes. As the farthest service 
distance of a maintenance point increases, the number of maintenance points required for construction gradually decreases. When the farthest protection distance of a maintenance point decreases, the number of maintenance points that must be built increases faster. Conversely, the greater the farthest protection distance of a maintenance point, the more slowly the number of required maintenance points is reduced.

It is worth pointing out the following:

Both performance experiments suffer from the problem of unevenly distributed maintenance points; future research should emphasize the causes of this phenomenon and whether it is the result of a change in the radius of coverage or of the principles or performance of the algorithm.

In actual scenarios in real-world practice, maintenance networks for urban rail transit systems also have to consider other technical and professional requirements. Future research should consider this design problem by taking professional maintenance into account to reach a more practical solution.

\section{Data Availability}

The data used to support the findings of this study are included within the article.

\section{Conflicts of Interest}

The authors declare no conflicts of interest.

\section{Authors' Contributions}

Y. J. designed the research methods; B. S. wrote the manuscript; L. Z. collected the data; W. C. analyzed the data; X. R. drew the figures; and $\mathrm{X}$. $\mathrm{M}$. edited and revised the manuscript.

\section{Acknowledgments}

This research was funded by the National Natural Science Foundation of China (grant number 51308249), the Special Research Fund for Doctoral Programs (grant number 20130061110008), and the Special Project for Industrial Technology Research and Development of Jilin Province (grant number 2015Y044), and it was supported by the Humanities and Social Science Research Program of the Ministry of Education in China (grant number 16XJCZH002) and the Fundamental Research Funds for the Central Universities (grant numbers 310823170657 and 300102238501).

\section{References}

[1] S. Dantrakul, C. Likasiri, and R. Pongvuthithum, "Applied $\mathrm{p}$-median and $\mathrm{p}$-center algorithms for facility location problems," Expert Systems with Applications, vol. 41, no. 8, pp. 3596-3604, 2014.

[2] C. Upchurch and M. Kuby, "Comparing the p-median and flow-refueling models for locating alternative-fuel stations,"
Journal of Transport Geography, vol. 18, no. 6, pp. 750-758, 2010.

[3] O. Berman and D. Krass, "The generalized maximal covering location problem," Computers \& Operations Research, vol. 29, no. 6 , pp. 563-581, 2002.

[4] A. Shariat-Mohaymany, M. Babaei, S. Moadi, and S. M. Amiripour, "Linear upper-bound unavailability set covering models for locating ambulances: application to Tehran rural roads," European Journal of Operational Research, vol. 221, no. 1, pp. 263-272, 2012.

[5] A. Başar, B. Çatay, and T. Ünlüyurt, "A taxonomy for emergency service station location problem," Optimization Letters, vol. 6, pp. 1147-1160, 2012.

[6] R. Z. Farahani, N. Asgari, N. Heidari, M. Hosseininia, and M. Goh, "Covering problems in facility location: a review," Computers \& Industrial Engineering, vol. 62, no. 1, pp. 368407, 2012.

[7] O. Berman, Z. Drezner, and D. Krass, "Generalized coverage: new developments in covering location models," Computers \& Operations Research, vol. 37, no. 10, pp. 1675-1687, 2010.

[8] R. Bai, N. Xue, J. Chen, and G. W. Roberts, "A set-covering model for a bidirectional multi-shift full truckload vehicle routing problem," Transportation Research Part B: Methodological, vol. 79, pp. 134-148, 2015.

[9] M. R. Akella, R. Batta, E. M. Delmelle, P. A. Rogerson, A. Blatt, and G. Wilson, "Base station location and channel allocation in a cellular network with emergency coverage requirements," European Journal of Operational Research, vol. 164, no. 2, pp. 301-323, 2005.

[10] O. Berman, Z. Drezner, D. Krass, and G. O. Wesolowsky, “The variable radius covering problem," European Journal of Operational Research, vol. 196, no. 2, pp. 516-525, 2009.

[11] M. Bashiri and F. Fotuhi, "A cost-based set-covering locationallocation problem with unknown covering radius," in Proceedings of 2009 IEEE International Conference on Industrial Engineering and Engineering Management, October 2009, Beijing, China.

[12] S. Davari, M. H. Fazel Zarandi, and I. Burhan Turksen, "A greedy variable neighborhood search heuristic for the maximal covering location problem with fuzzy coverage radii," Knowledge-Based Systems, vol. 41, pp. 68-76, 2013.

[13] D. Simon, "Biogeography-based optimization," IEEE Transactions on Evolutionary Computation, vol. 12, no. 6, pp. 702-713, 2008.

[14] D. Simon, M. G. H. Omran, and M. Clerc, "Linearized biogeography-based optimization with re-initialization and local search," Information Sciences, vol. 267, pp. 140-157, 2014.

[15] M. Ergezer, D. Simon, and D. Du, "Oppositional biogeography-based optimization," in Proceedings of 2009 IEEE International Conference on Systems, Man and Cybernetics, San Antonio,TX, USA, October 2009.

[16] H. Ma and D. Simon, "Blended biogeography-based optimization for constrained optimization," Engineering Applications of Artificial Intelligence, vol. 24, no. 3, pp. 517-525, 2011.

[17] R. Shukla and D. Singh, "Experimentation investigation of abrasive water jet machining parameters using Taguchi and Evolutionary optimization techniques," Swarm and Evolutionary Computation, vol. 32, pp. 167-183, 2017. 